\title{
Gary Liney (ed): MRI from A to Z: a Definitive Guide for Medical Professionals
}

\author{
Springer-Verlag London Limited, 2010, ISBN: 978-1-84996-135-6
}

\author{
Mariarosaria Prisco $\cdot$ Luigi Mansi
}

Published online: 27 February 2013

(C) Springer-Verlag Berlin Heidelberg 2013

'MRI from A to Z: a Definitive Guide for Medical Professionals' is the second edition, much larger and significantly updated, of a book first published in 2005. It consists of 385 pages, containing more than 1,300 entries and about 100 illustrations. The publication is a compendium of terms used in magnetic resonance (MR) presented in alphabetical order from A to Z. Gary Liney is an expert physicist who has worked for many years in the field of MR imaging (MRI) and spectroscopy (MRS) both at the clinical and academic level. At present, he is the Principal Physicist for Oncology Imaging and Radiotherapy Physics at the Queen's Centre for Oncology, Castle Hill Hospital, Cottingham, UK.

The publication is a reflection of the rapid growth of the field of MRI that continues to find new areas of application and is increasingly used in combination with other imaging modalities, both in integrated diagnostic pathways and in hybrid machines. The vocabulary necessary to keep up with this evolution has increased exponentially. Therefore, compared to the previous edition, the amount of text has increased by approximately $80 \%$, containing more figures and including new achievements, and each item has been re-evaluated and updated where necessary. The book is a pocket-friendly manual, easy to consult, that provides a wide range of theo- retical and practical information through the analysis of the specific terminology used in MRI. Each definition is explained in a concise, clear and comprehensive way, often with references, charts and figures that better explain the meaning and provide the reader with more insight. The length of each term frequently occupies more than a few lines, sometimes requiring a wider explanation, but never reaching the space of a page. This is a very clever didactic approach, because all the definitions are clear but are not too complex to be understood. Despite the fact that a good basic knowledge of the complex subject of MRI is required, the explanations are simple and easy to understand.

Therefore this is a very practical text, representing an invaluable reference tool for radiologists, physicists, technologists and trainees who work with MRI and, more generally, in diagnostic imaging. The publication may be of fundamental utility in daily clinical practice especially for those who want a quick understanding of key concepts. This group includes people working in nuclear medicine, among whom there is certainly a growing interest in the field, and who may seek to interact better with radiologists and be ready to understand and utilize new diagnostic pathways and new hybrid machines, such as PET/MRI.
M. Prisco $\cdot$ L. Mansi $(\bowtie)$

Second University of Naples, Naples, Italy

e-mail: luigi.mansi@unina2.it 